\title{
Therapeutic drug monitoring of levetiracetam in daily clinical practice: high-performance liquid chromatography versus immunoassay
}

\author{
Maria Mendoza Aguilera, María Dolores Bellés Medall, Tamara Álvarez Martín, \\ Óscar Pascual Marmaneu, Carla Liñana Granell @ — , Raúl Ferrando Piqueres
}

Department of Clinical Pharmacy, Hospital General Universitario de Castellón, Castellon, Spain

\section{Correspondence to}

Maria Mendoza Aguilera, Department of Clinical Pharmacy, Hospital General Universitario de Castellón, Castellon 12004, Spain; mendoza_maria88@hotmail. com

Received 14 May 2018 Revised 14 September 2018 Accepted 25 September 2018 Published Online First 20 October 2018

EAHP Statement 4: Clinical Pharmacy Services.

\section{Check for updates}

(C) European Association of Hospital Pharmacists 2020. No commercial re-use. See rights and permissions. Published by BMJ.

To cite: Mendoza Aguilera M, Bellés Medall MD, Álvarez Martín T, et al. Eur J Hosp Pharm 2020;27:e2-e6.

\section{ABSTRACT \\ Objectives Although levetiracetam presents an easy} dosing and tolerability, therapeutic drug monitoring may be recommended in certain situations. Measurement of levetiracetam in serum plasma is commonly done by high performance liquid chromatography (HPLC). After ARK Diagnostics marketed an enzyme immunoassay (IA) for levetiracetam in serum or plasma, automated determinations are possible. In this study, the performance of this immunoassay and the impact of automation on the follow-up in patients treated with levetiracetam is evaluated. We also detected those subpopulations of patients who may benefit the most from this therapeutic drug monitoring.

Methods Samples from 50 outpatients diagnosed with epilepsy and treated with levetiracetam were collected. This new IA was performed on the Architect c4000 analyser and compared with the HPLC. Then, a retrospective observational study that included serum samples of levetiracetam for 24 months, was conducted to evaluate the impact of automattion and the influence of some variables (age, sex, renal function, and coadministration of valproic acid and glucuronidationinducing drugs) in levetiracetam apparent oral clearance (CLp/F) by a multivariate linear regression.

Results The mean high-performance liquid chromatography quantified concentration (CpHPLC) was $18.43 \mathrm{mcg} / \mathrm{mL}$ (95\% Cl: 15.48 to 21.39) and immunoassay concentration (CpEl) was $18.35 \mathrm{mcg} / \mathrm{mL}$ (95\% Cl: 15.20 to 21.50) ( $P=0.861)$. The Pearson's linear correlation coefficient obtained in the analysis was $r^{2}=0.88$, according to the following equation: $\mathrm{CpHPLC}=-0.29+1.01 \mathrm{CpEl}$. The intraclass correlation coefficient was 0.95 (95\% Cl: 0.91 to 0.97). After IA implementation, the number of levetiracetam determinations increased in $76.27 \%$. The median of Clp/F was higher $(P<0.001)$ in inducers (4.36 L/h; IQR:3.29-5.44) and lower $(\mathrm{P}<0.001)$ in glomerular filtration rate $(\mathrm{GFR})$ $<60 \mathrm{~mL} / \mathrm{min}$ (2.7L/h; IQR: 0.58-3.85).

Conclusions The Ark method performed on the Architect is fully acceptable and can be used routinely to measure levetiracetam plasmatic concentration levels. It has demonstrated the need for closer monitoring in patients with renal failure or co-administration of glucuronidation-inducing drugs.

\section{INTRODUCTION}

Levetiracetam is an anticonvulsant whose mechanism of action is thought to involve binding of the synaptic vesicle protein SV2A, which is related to neurotransmitter vesicle exocytosis. ${ }^{1}$
This antiepileptic drug is available as monotherapy for epilepsy in partial seizures, and as an adjunctive therapy for partial, myoclonic and primary generalised tonic-clonic seizures.

Levetiracetam shows a rapid and almost complete absorption after oral administration, although the oral absorption rate is slowed down by co-ingestion with food. ${ }^{2}$ Pharmacokinetics are linear and time-invariant, with low inter- and intrasubject variability. Levetiracetam does not significantly bound to proteins and it is renally excreted (66\% unchanged and 24\% as the major metabolite, which is pharmacologically inactive). Drug interactions are uncommon because levetiracetam is not metabolised by the liver. ${ }^{34}$

Like many drugs, pharmacokinetics of levetiracetam differs between children and adults. Pharmacokinetic processes of absorption, distribution, metabolism and excretion undergo changes due to growth and development. The serum half-life of this antiepileptic drug is different in neonates (16-18 hours) and adults (6-8 hours) ${ }^{3}$ and the increase in clearance in children implies a higher dose $\mathrm{mg} / \mathrm{kg}$ in patients up to 12 years' old. ${ }^{5}$ Moreover, a significant $60 \%$ decrease in serum levetiracetam concentrations during pregnancy is observed. $^{36}$

Although levetiracetam has an easy dosing and tolerability, therapeutic drug monitoring may be indicated in some situations (suspicion of noncompliance, pregnancy, children dosage, renal failure and ineffectiveness with high doses). In patients with renal failure, the daily dose of this anticonvulsant should be adjusted and drug monitoring is recommended. ${ }^{3}$

The therapeutic range of levetiracetam considered was a trough level of 12 to $46 \mu \mathrm{g} / \mathrm{mL}^{17}$

Measurement of levetiracetam in serum plasma is commonly done by high-performance liquid chromatography (HPLC). ${ }^{8-10}$ Although HPLC is an accurate technique, as iis proved by several studies, ${ }^{11} 12$ it involves a very complex and highly time-consuming methodology, which renders it difficult to use in daily clinical practice. Samples are not analysed immediately, on the contrary they are saved and analysed all together in the day of the week agreed. In addition, it is not possible to perform urgent determinations. After an increase in demand for levetiracetam monitoring, as part of the routine and also as urgent petitions, we had to find and evaluate an alternative analytical technique. 
ARK Diagnostics has recently merchandised an immunoassay for levetiracetam in serum or plasma which makes an automated determination possible. But first of all, the performance of this immunoassay must be evaluated using HPLC-ultraviolet (UV) as the reference method.

The main objective of the study was to validate IA as a new analytical technique by a concordance study between IA and HPLC-UV (taken as the gold standard). Secondary objectives were, first, to evaluate the impact of the automation of the technique in clinical practice by assessing the chance in the turnaround and the number of levetiracetam determinations, and, second to evaluate the influence of different covariables (age, sex, renal function, co-administration with valproic acid and glucuronidation-inducing drugs) on apparent oral clearance $(\mathrm{CLp} / \mathrm{F})$ of levetiracetam in order to identify those subgroups in which close monitoring should be carried out.

\section{MATERIAL AND METHODS \\ Patient samples}

Fifty serum specimens were gathered for the concordance study. Blood samples were collected in BD Vacutainer Plus Plastic Serum tubes. They were centrifuged $(1500 \mathrm{x} \mathrm{g}$ for $10 \mathrm{~min}$ at $4^{\circ} \mathrm{C}$ ), divided into two aliquots and then stored at $-20^{\circ} \mathrm{C}$ until their analysis by both methods: HPLC and immunoassay.

\section{Instruments and methods}

The chromatographic system included an automatic injector (Hitachi Autosampler L-220), a pump (Hitachi L-6200A), an ultraviolet absorption detector (Merk Hitachi L4250) and Ez Chrom Elite v 3.1.7 integrator (Pleasanton, USA). At the stationary phase it was used at LiChroCART 250-4.6 RP-18 endcapped $25 \mathrm{~cm}$ column (international particle diameter $5 \mu \mathrm{m}$ ). The mobile phase consisted of a mixture of potassium dihydrogen phosphate buffer $(50 \mathrm{~mm}, \mathrm{PH}=4.5)$ and acetonitrile/ methanol (3/1) $(65: 35, \mathrm{v} / \mathrm{v})$, and was delivered at a flow rate of $1 \mathrm{~mL} / \mathrm{min}$.

It was included as a five-point calibration line with a range of $5 \mu \mathrm{g} / \mathrm{mL}$ to $80 \mu \mathrm{g} / \mathrm{mL}$. Linearity was evaluated by calculation of a regression line by the least squares method, between the concentrations and the areas of the chromatogram. Samples of $150 \mu \mathrm{L}$ were mixed with $300 \mu \mathrm{L}$ of precipitating solution containing zinc sulphate, and after centrifugation, it was introduced into the injector.

ARK levetiracetam assay is a homogeneous immunoassay based on competition between the drug contained in the specimen and some levetiracetam labelled with glucose-6-phosphate dehydrogenase (G6PDH) enzyme which binds to the antibody reagent. When the binding occurs, enzyme activity decreases. Samples with the presence of levetiracetam show an increase in the enzyme activity directly proportional to the drug concentration. The enzyme reaction results in the conversion of cofactor $\mathrm{NAD}$ to $\mathrm{NADH}$, which is measured spectrophotometrically at $340 \mathrm{~nm}$ (linearity range: $2.0-100 \mu \mathrm{g} / \mathrm{mL}$ ).

The ARK levetiracetam assay was performed on an Architect c4000 automated chemistry analyser, marketed by Abbott Diagnostics Illinois, (LaUSA). ARK provided six calibration standards at concentrations of $0,5.0,12.5,25.0,50.0$ and $100.0 \mu \mathrm{g} / \mathrm{mL}$, which were analysed by duplicate. Linearity of the method was considered acceptable by the manufacturer from 2.0 to $100.0 \mu \mathrm{g} /$ $\mathrm{mL}$.

\section{Performance verification}

Both HPLC and ARK levetiracetam assay have been validated in previous studies. ${ }^{5-7}$ However, analytical performances declared by manufacturers were verified before beginning the study. Commercially controlled samples supplied by ARK at levels of $7.5,29.4$ and $73.4 \mu \mathrm{g} / \mathrm{mL}$ were run during each analysis with both methods.

The assay was considered acceptable if intraassay and interassay precision (CV) should not exceed 15\% with accuracy placed within $\pm 15 \%$.

\section{Statistical evaluation}

Concordance was evaluated through intraclass correlation coefficient (ICC), and Lin's concordance correlation coefficient (CCC) calculated by Passing Bablok regression, Bland- Altman Plot and cumulative distribution plot, where a acceptable limit of $3.5 \mu \mathrm{g} / \mathrm{mL}$ was defined a priori. Values were expressed as mean (95\% CI).

Statistical analysis was performed with SPSS (V19.0; IBM SPSS Statics; Chicago, Illinois, USA).

A difference was considered statistically significant at $\mathrm{P}<0.05$.

\section{Impact of automation in clinical practice}

We also conducted a retrospective observational study that included serum samples of levetiracetam of 36 months: 12 months before and 24 months after the implementation of IA. The turnaround time between the two periods was compared. The number and percentage of levetiracetam determinations of total antiepileptic determinations with HPLC and IA were analysed.

\section{Influence of different covariables on the apparent oral clearance}

Variables such as age, sex, pregnancy, glomerular filtration (GF), co-administration of valproic acid and association with glucuronidation-inducing drugs (such as carbamazepine or phenytoin) were analysed. The influence of these variables in levetiracetam metabolism was evaluated using the apparent oral clearance $(\mathrm{CLp} / \mathrm{F})$. A multivariate linear regression was designed to determine which cofactors could be associated with CLp/F.

$$
\frac{C L}{F}\left[\frac{L}{b}\right]=\frac{L T G_{-} \text {dose }[\mathrm{mg} / \text { day }] / L T G_{-} \text {dose_interval }[\mathrm{b}]}{\text { plasma_concentration }[\mathrm{mg} / \mathrm{L}]}
$$

\section{RESULTS}

\section{Validation study}

Both methods showed good precision when they were evaluated with commercial control samples $(7.5,29.4$ and $73.4 \mu \mathrm{g} / \mathrm{mL})$. The observed CV at the low-quality control was $3.48 \%$, with a mean of $7.75 \mu \mathrm{g} / \mathrm{mL}$. At the medium quality control level, a CV of $3.04 \%$ was observed, with a mean of 30.38 . At the highquality control level, the observed CV was $2.42 \%$ with a mean of $77.04 \mu \mathrm{g} / \mathrm{mL}$.

Fifty serum specimens were analysed by both methods: HPLC and IA. The average concentration quantified with HPLC was $18.35 \mu \mathrm{g} / \mathrm{mL}$ (95\% CI, 15.20 to 21.50 ) and with the Architect c 4000 was $17.78 \mu \mathrm{g} / \mathrm{mL}$ (95\% CI, 14.82 to 20.74 ).

Both methods were compared by a regression analysis (slope with $95 \% \mathrm{CI}$, intercept with $95 \% \mathrm{CI}$ ), which showed an intercept of 1.01 (95\% CI, -2.33 to $1.78, \mathrm{P}=0.79)$ and a slope of 0.29 (95\% CI, 0.21 to $0.91, \mathrm{P}<0.001$ ) according to the equation $\mathrm{CpHPLC}=-0.29+1.01 \mathrm{CpARK}\left(\mathrm{r}^{2}=0.88, \mathrm{P}<0.001\right)$. (figure 1$)$

The result of CCI (0.95) showed a very good agreement $(>90)$ between both methods. The CCC, stricter than the previous one, 


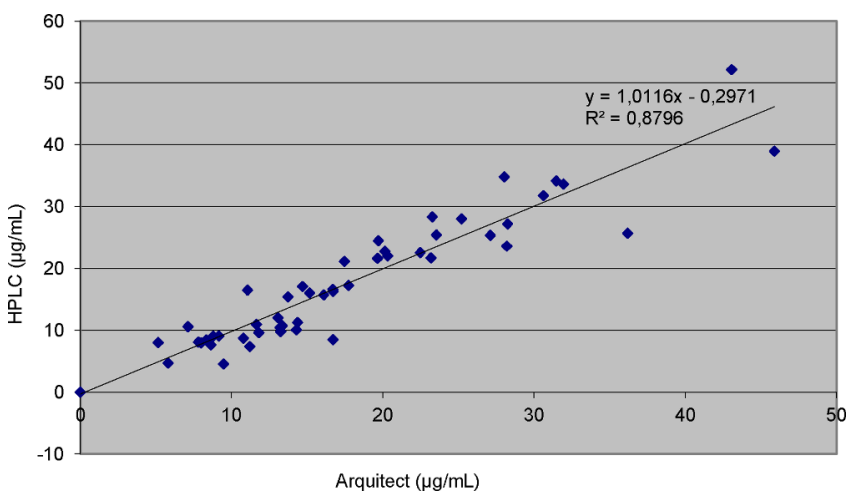

Figure 1 The regression plot shows the linearity of the HPLC/ARK assay.

showed a good agreement (0.95-0.99), with a result of 0.97 . The Passing Bablok regression line obtained an intercept of 1.74 (95\% CI, -0.01 to 3.01) and a slope of 0.90 (95\% CI, 0.83 to 1.09 ) which suggested a non-significantly constant systematic error and non-significantly proportional systematic error.

The Bland-Altman plot showed a systematic bias of 0.076 $\mathrm{mcg} / \mathrm{mL}(95 \% \mathrm{CI},-7.05$ to 7.19$)$ and a random error of 15.34 (figure 2).

Another method for agreement evaluation is the cumulative distribution plot, which shows the absolute differences between two measurements versus the proportion of cases where discrepancies match, at least, with the observed differences. A concordance limit of $3.5 \mu \mathrm{g} / \mathrm{mL}$ was established (the therapeutic range we used was $12-46 \mu \mathrm{g} / \mathrm{mL}$ ). Seventy-four per cent of the difference between the two methods was less than $3.5 \mu \mathrm{g} / \mathrm{mL}$ (figure 3).

If we stratify the results between those below the therapeutic range $(<12 \mu \mathrm{g} / \mathrm{mL})$ and those within the therapeutic range $(12-46 \mu \mathrm{g} / \mathrm{mL})$, the mean deviation percentages of levetiracetam with the immunoassay method compared with HPLC was $12.46 \%(6.09-21.32)$ in the samples within the therapeutic range, and $18.39 \%(2.49-41.62)$ in the samples below the therapeutic range.

\section{Impact of automation in clinical practice}

We compared the turnaround time before and after IA implementation. Because of the high complexity of the HPLC technique, the samples were centrifuged and stored at $-20^{\circ} \mathrm{C}$ for the next batch by a chromatography-based method, obtaining a turnaround time of 7 days approximately. With this new IA method, samples can be analysed immediately, obtaining a response and elaborating a pharmacokinetic report in $60 \mathrm{~min}$ from the reception of the sample in the pharmacokinetic unit.

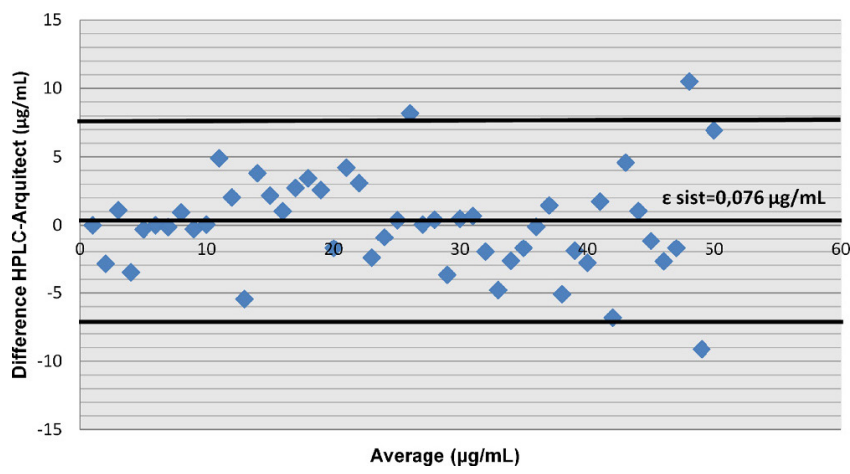

Figure 2 Bland-Altman analysis of the comparison between Architect and HPLC method.

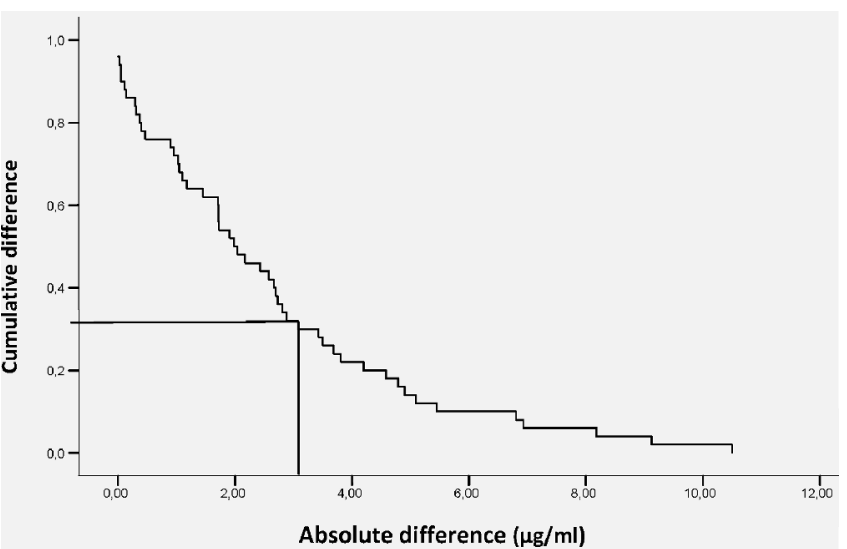

Figure 3 Cumulative distribution plot.

After the first year of IA implementation, the number of levetiracetam determinations increased by $76.27 \%$, representing $13.88 \%$ of the total antiepileptic determinations. A total of 236 samples were analysed, 39 of them considered as urgent (16.5\%).

The second year, the number of determinations raised to 329 , representing $19.18 \%$ of the total of antiepileptic drugs. The number of urgent determinations also increased, representing $25 \%$ of the determinations.

\section{Identify the subgroups in which close monitoring must be carried out}

In order to identify the subgroups which may benefit the most from pharmacokinetic monitoring, patients were stratified according to different cofactors. Twenty-two per cent of patients were on valproic treatment, $19 \%$ presented renal failure (glomerular filtration lower than $60 \mathrm{~mL} / \mathrm{min}$ ), 14\% had an antiepileptic UGT inducer, 3\% were under 14 years' old and the rest $(42 \%)$ did not have any associated cofactor.

A different pharmacokinetic pattern was observed in the different population subgroups: patients younger than 14 years showed greater clearance of the drug, being approximately $50 \%$ of the patients, below the therapeutic range. In addition, $40 \%$ of patients treated with an enzyme inducer also presented infratherapeutic levels. On the other hand, $24 \%$ of the patients with glomerular filtration lower than $60 \mathrm{~mL} / \mathrm{min}$, obtained levels higher than $46 \mathrm{mg} / \mathrm{mL}$ (figure 4 ).

Multivariate analysis associated UGT inducers $(\mathrm{P}<0.001)$ with increased $\mathrm{Clp} / \mathrm{F}$, whereas glomerular filtration lower than $60 \mathrm{~mL} /$ $\min (\mathrm{P}<0.001)$ was associated with a reduction in $\mathrm{Clp} / \mathrm{F}$. The

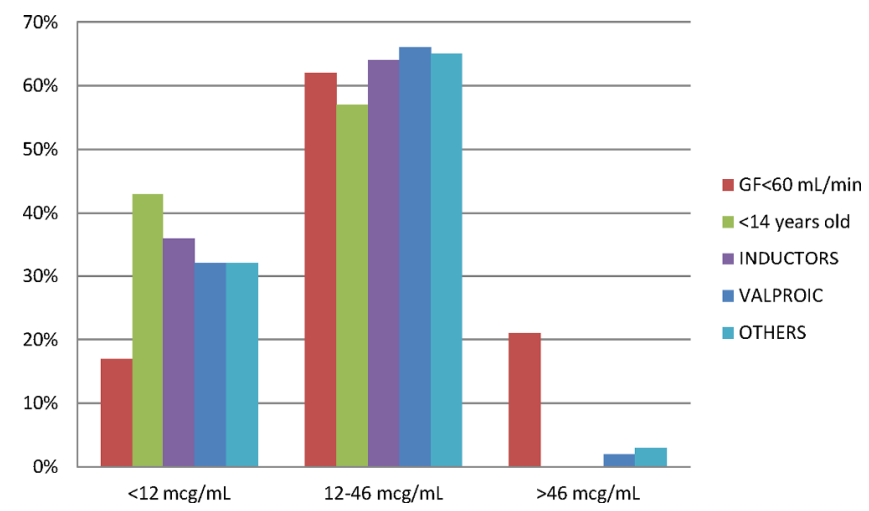

Figure 4 The median of $\mathrm{Clp} / \mathrm{F}$ in the different population groups analysed. 
Table 1 Multivariate regression

\begin{tabular}{llllll}
\hline Cl/F (L/h) & Coef. & Std. err. & $\mathbf{t}$ & $\mathbf{p}$ & $\mathbf{9 5 \%} \mathrm{Cl}$ \\
\hline Valproic & -0.2778 & 0.4849 & -0.57 & 0.567 & -1.2323 to 0.6766 \\
GF $<60 \mathrm{~mL} / \mathrm{min}$ & -2.7165 & 0.5774 & -4.70 & $<0.001$ & -3.8529 to -0.5802 \\
Inductors & 4.3639 & 0.5470 & 7.98 & $<0.001$ & 3.2874 to 5.4405 \\
Age & -0.0132 & 0.0103 & -1.29 & 0.200 & -0.0336 to 0.0070 \\
Sex & 0.3336 & 0.4094 & 0.81 & 0.416 & -0.4723 to 1.1395 \\
Constants & 4.6194 & 0.8328 & 5.55 & $<0.001$ & 2.9802 to 6.2584 \\
\hline
\end{tabular}

highest pharmacokinetic follow-up was in patients co-treated with inducers, with a ratio of 2.3 determinations per patient (table 1).

\section{DISCUSSION}

Implementation of an immunoassay for levetiracetam monitoring using automated instrumentation represents many advantages in comparison with HPLC, such as shorter turnaround time and simpler sample preparation.

After having revised the literature we found other studies evaluating ARK levetiracetam assay performed on a Siemens ADVIA automated chemistry analyser and on the ThermoFisher Scientific Inc. analyser, ${ }^{11} 12$ but we did not find any information concerning the Architect c4000 automated chemistry analyser.

In these two articles a good concordance was found between ARKlevetiracetam assay and HPLC. The first one used 59 samples and showed a correlation coefficient of 0.9962 , a linear regression slope of 0.98 and an intercept of 0.61 with a mean bias of $0.04 \%$. Similarly, the article of Bianchi et al ${ }^{12}$ used 63 samples and obtained a minimum systematic bias of $1.0 \mu \mathrm{g} / \mathrm{mL}$ (95\% CI 0.32 to 1.69 ) with $95 \%$ of the HPLC-Ark differences ranging from $-4.3 \mu \mathrm{g} / \mathrm{mL}(95 \% \mathrm{CI}-5.52$ to 3.16$)$ and $6.3 \mu \mathrm{g} /$ $\mathrm{mL}$ (95\% CI 5.16 to 7.52). They also calculated a Passing-Bablok regression analysis that showed a non-significant intercept of 0.16 and a slope marginally significantly different from unity of 0.95 (95\% CI, 0.90 to 0.99 ), which suggested some minimum proportional systematic error.

Concordance analysis is needed to establish the validity of a new diagnostic measuring or rating technique, or to demonstrate the near equivalence of multiple measuring or rating techniques. Erroneous approaches to concordance analysis can lead to false conclusions. For this reason, the concordance between the ARK levetiracetam assay performed on the Architect c4000 automated chemistry analyser and the reference method (HPLC) has been studied.

A linear relationship was demonstrated between 0 and $52 \mu \mathrm{g} /$ $\mathrm{mL}$, with Pearson's regression off $0.88(\mathrm{P}<0.001)$. The linearity only measures the intensity of the association between both variables, but it does not inform about the concordance.

To evaluate the concordance, and according to the characteristics of the variables (continuous data measures), we decided to choose calculating the CCI and the CCC, and interpreting them in a Bland-Altman plot and a cumulative distribution plot.

The CCI is a measure of the reliability of measurements or ratings. The result of 0.95 showed almost perfect agreement. However, some authors criticised the CCI as a measure of agreement among observers because it cannot measure the lack of accuracy (ie, difference of means) between observers' measures.

CCC obtained was 0.93 , which indicates a moderate agreement. This coefficient is more robust and does not change significantly when the assumptions of normality are not met.
Accordingly, the Bland-Altman analysis showed a minimum systematic error of $0.076 \mu \mathrm{g} / \mathrm{mL}$ with $95 \%$ of the HPLC-Ark different ranging from -7.05 to $7.19 \mu \mathrm{g} / \mathrm{mL}$ and a random error of 15.34. However, the plot showed that the spread of the differences remained consistent across the range of concentration.

To continue with the evaluation, a 'Kaplan-Meier graph' was constructed, where the horizontal axis represented the absolute difference between two measurements for each subject and the vertical axis represented the proportion of cases where discrepancies equal, at least, each of the differences. Seventy-four per cent of the difference between the two measurements was less than $3.5 \mu \mathrm{g} / \mathrm{mL}$.

The demand for urgent determinations of levetiracetam is increasing for several reasons: a change in the profile of use of antiepileptics, an increase in the prescription of the newest antiepileptics (levetiracetam, lamotrigine) and a decrease in the use of the former ones, such as phenytoin. Including therapeutic drug monitoring of the new antiepileptics for common clinical practice has proven a high value when making a therapeutic decision.

This technique, much simpler and faster, has allowed us to analyse an increasing amount of urgent samples and to offer levetiracetam level results when needed.

In addition, it has made possible a closer monitoring of the patients, especially those who need it most: subpopulations co-treated with antiepileptic inducers and reduced glomerular filtration.

However, there are other subgroups of patients which have different pharmacokinetic behaviours, such as pregnant women and the paediatric population.

In a retrospective $\operatorname{study}^{13}(\mathrm{n}=21)$, the serum concentration/ dose ratios were analysed in pregnant women with seizures treated with levetiracetam. The serum concentration/dose ratios of levetiracetam gradually decreased during pregnancy, by as much as $50 \%$ during the third trimester, and returned to baseline values postpartum. In our case, pregnancy could not be assessed as a cofactor because there was not any patient in this situation during our study period. However, we are beginning to monitor more pregnant women treated with levetiracetam

On the other hand, it is expected that there will be an increase in $\mathrm{Clp} / \mathrm{F}$ in the paediatric population. According to this study and its pivotal trials ${ }^{2}$ and after a single oral dose of levetiracetam $(20 \mathrm{mg} / \mathrm{kg} / \mathrm{day})$, elimination half-life was shorter (5.3 hours) among paediatric patients (1 month to less than 4 years) compared with adult subjectss (7.2 hours). In another study ${ }^{6}$ of 15 children and 14 women with epilepsy treated with adjunctive $(n=8)$ or monotherapy $(n=6)$ levetiracetam 2000 to $6000 \mathrm{mg} /$ day, the mean elimination $\mathrm{t}(1 / 2)$ of levetiracetam in neonates was 18 hours. Due to the small sample size (3\%) of the present study, it has not been possible to obtain results in the multivariate analysis concerning this population group. 


\section{CONCLUSION}

To conclude, the Ark method on the Architect c4000 is proven to be acceptable and reliable, and can be used routinely to measure levetiracetam plasmatic concentrations. Compared with chromatography-based methods, this assay reduced the turnaround time and simplified sample preparation in daily clinical practice, increasing the follow-up of patients treated with levetiracetam.

\section{What this paper adds}

\section{What is already known on this subject}

- Measurement of levetiracetam in serum plasma is commonly done by high-performance liquid chromatography (HPLC).

- HPLC is an exact technique but has an elevated turnaround time. For this reason it is difficult to use in daily clinical practice.

- In the literature we found other studies evaluating the ARK Levetiracetam Assay performed on a Siemens ADVIA automated chemistry analyser and in the ThermoFisher Scientific Inc analyser, but we did not find any information with the Architect c4000 automated chemistry analyser.

\section{What this study adds}

- The Ark method on the Architect c4000 is acceptable and can be used routinely to measure levetiracetam plasmatic concentrations

- Compared with chromatography-based methods, this assay reduced turnaround time and simplified the preparation in daily clinical practice

- Since the implementation of the technique, more determinations have been performed.In addition, it has allowed a closer monitoring of the patients, above all those who need it most: co-treated with inducers and reduced glomerular filtration.
Funding The authors have not declared a specific grant for this research from any funding agency in the public, commercial or not-for-profit sectors.

Competing interests None declared.

Patient consent Not required.

Provenance and peer review Not commissioned; externally peer reviewed.

ORCID iD

Carla Liñana Granell http://orcid.org/0000-0002-5500-9307

\section{REFERENCES}

1 Krasowski MD. Therapeutic drug monitoring of the newer anti-epilepsy medications. Pharmaceuticals 2010;3:1909-35.

2 Keppra Data sheet. http://www.ema.europa.eu/docs/es_ES/document_library/ (accessed 15 Jan 2018).

3 Patsalos PN, Berry DJ, Bourgeois BF, et al. Antiepileptic drugs - best practice guidelines for therapeutic drug monitoring: a position paper by the subcommission on therapeutic drug monitoring, ILAE Commission on Therapeutic Strategies. Epilepsia 2008;49:1239-76

4 Patsalos PN. Antiepileptic Drug Interactions: A Clinical Guide. Guildford: Clarius Press, 2005.

5 O'Hara K. Paediatric pharmacokinetics and drug doses. Aust Prescr 2016;39:208-10.

6 Tomson T, Palm R, Källén K, et al. Pharmacokinetics of levetiracetam during pregnancy, delivery, in the neonatal period, and lactation. Epilepsia 2007:48:1111-6.

7 Aldaz A, Ferriols R, Aumente $D$, et al. Monitorización farmacocinética de antiepilépticos. Farmacia Hospitalaria 2011;35:326-39.

8 Baldelli S, Cattaneo D, Giodini L, et al. Development and validation of a HPLC-UV method for the quantification of antiepileptic drugs in dried plasma spots. Clin Chem Lab Med 2015;53:435-44.

9 Shihabi ZK, Oles K, Hinsdale M. Analysis of the antiepileptic drug keppra by capillary electrophoresis. J Chromatogr A 2003;1004:9-12.

10 Pucci V, Bugamelli F, Mandrioli R, et al. High-performance liquid chromatographic determination of Levetiracetam in human plasma: comparison of different sample clean-up procedures. Biomed Chromatogr 2004;18:37-44.

11 Reineks EZ, Lawson SE, Lembright KE, et al. Performance characteristics of a new levetiracetam immunoassay and method comparison with a high-performance liquid chromatography method. Ther Drug Monit 2011;33:124-7.

12 Bianchi V, Arfini C, Vidali M. Therapeutic drug monitoring of levetiracetam: comparison of a novel immunoassay with an HPLC method. Ther Drug Monit 2014;36:681-5.

13 Westin AA, Reimers A, Helde G, et al. Serum concentration/dose ratio of levetiracetam before, during and after pregnancy. Seizure 2008;17:192-8. 